\title{
Exotics at the LHC
}

\section{Daniele del $\mathbf{R e}^{* \dagger}$}

Sapienza Università and INFN Sezione Roma

E-mail: daniele.delrearomal.infn.it

LHC has worked beautifully and provided more than $100 \mathrm{fb}^{-1}$ at $13 \mathrm{TeV}$. Thanks to this enormous statistics of p-p collisions, LHC experiments have been able to explore several different new physics scenarios. We present the most recent results of the ATLAS, CMS, and LHCb experiments on the so-called Exotics searches for physics beyond the Standard Model.

The 39th International Conference on High Energy Physics (ICHEP2018)

4-11 July, 2018

Seoul, Korea

* Speaker.

${ }^{\dagger}$ on behalf of the ATLAS, CMS, and LHCb collaborations 


\section{Introduction}

The standard model (SM) of particle physics has proven extremely successful, but it still remains incomplete. There are several unanswered questions. Some examples are:

- why are there only three families of leptons and quarks?

- why are there four fundamental interactions and not one?

- why is force unification impossible even at very large energies

- why is only 5\% of matter made of ordinary SM particles? What is dark matter?

- why is the most massive particle "only" 200 times heavier than proton? Why is there a desert above $170 \mathrm{GeV}$ ?

There is a plethora of models and different search strategies performed at the LHC which are aimed to extend the SM and give answers to the questions above. Each of them introduces new particles and new physic processes to be searched for and tested at the experiments ([1, 2, 3]).

LHC has worked beautifully and provided, by July 2018, more than $100 \mathrm{fb}^{-1}$ of $13 \mathrm{TeV}$ proton-proton collisions and about $40 \mathrm{fb}^{-1}$ additional statistics should be collected before the end of Run 2. The large center of mass energy and luminosity obtained at the LHC give a unique opportunity to investigate the different extensions of the Standard Model. The only drawback of this large instantaneous luminosity is the number of concurrent collisions which increase the background and complicates the reconstruction (Fig. 1).
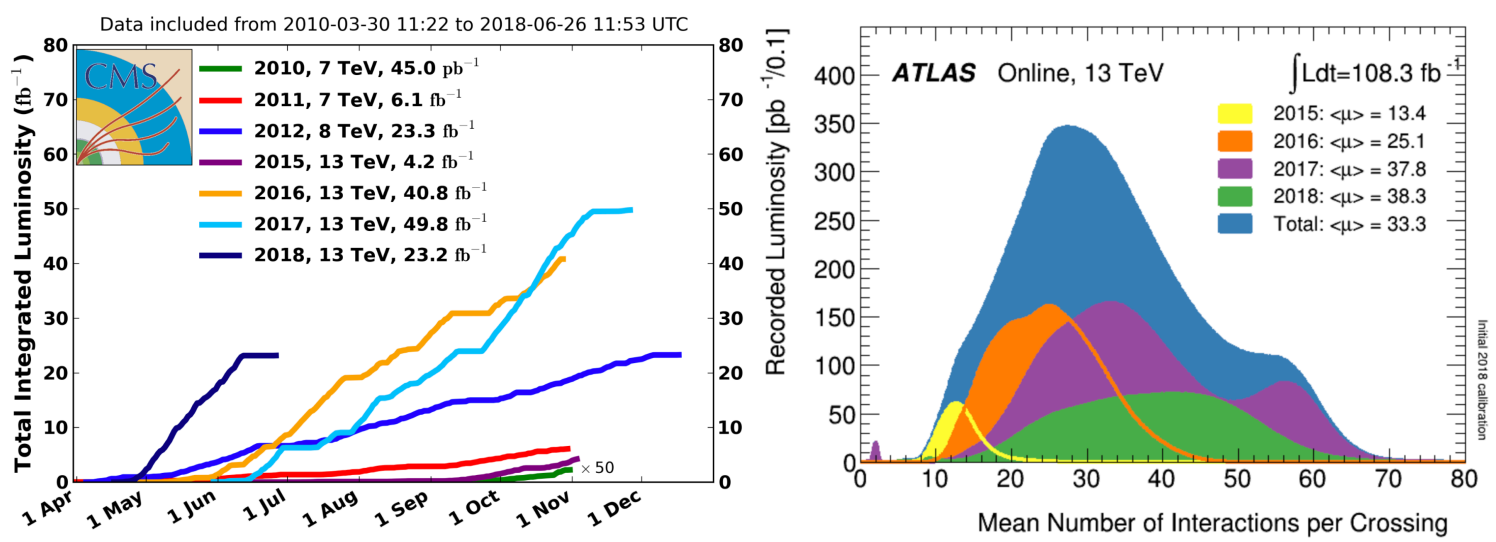

Figure 1: Luminosity recorded by the CMS experiment [4] (left) and number of interactions per crossing for the ATLAS experiment [5] (right) for the different years of data taking.

Due to space constraints, we cannot provide a comprehensive review of all exotics analyses performed at the LHC with $13 \mathrm{TeV}$ proton-proton collisions but rather a snapshot of the latest results, trying to divide them in terms of physics signatures and theory scenarios. The complete list of all public results from the three collaborations is available on the web for ATLAS [6], CMS [7], and $\mathrm{LHCb}$ [8]. In this review searches are grouped in four topics: heavy resonances, new leptons and quarks, long-lived particles, and dark matter. These topics correspond to what are usually called "Exotics" searches at the LHC. All SUSY searches, which represent another very large and comprehensive group of analyses, will be discussed in a different article. 


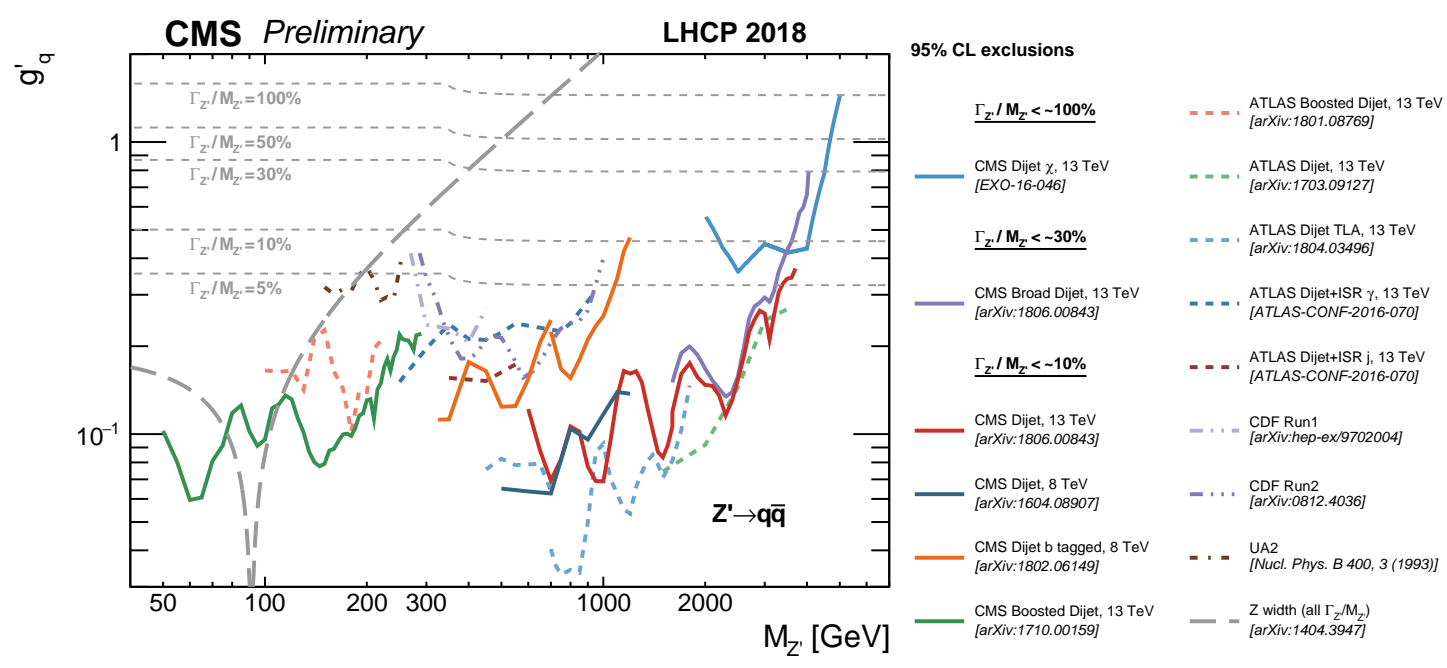

Figure 2: Limits on the universal coupling $g_{q}^{\prime}$ between a leptophobic Z' boson and quarks from various dijet analyses from CMS, ATLAS, CDF, and UA2 [9]. The limits are shown in solid lines, with the excluded area above the lines. The grey dashed lines show the $g_{q}^{\prime}$ values at fixed values of $\Gamma_{Z}^{\prime} / M_{Z}^{\prime}$.

\section{Resonances}

Many models predict the existence of heavy resonances that decay to a pair of standard model particles, as for example excited quarks, strings, new heavy bosons, gravitons, and dark matter. The signatures explored include basically all possible final states, combination of pairs of jets, leptons, photons, but also more complicated objects which can be also composite, like $\mathrm{W}$ and $\mathrm{Z}$ bosons and top quarks. Fully reconstructed resonances represent the simplest way to discover new particles since they offer striking and incontrovertible signatures based on the reconstructed invariant mass and small systematics in the background subtraction.

\subsection{Dijet}

The searches in dijet represent the best approach to search for extremely high masses, thanks to the large cross section involving final states with jets. The last big step in sensitivity happened in 2015, when the center of mass energy moved from 8 to $13 \mathrm{TeV}$. Since no more large changes in energy will take place in the near future, the sensitivity is expected to grow slowly, proportionally to the square root of the luminosity. For this reason, experiments have worked very hard to boost the sensitivity on the other side of the spectrum, at smaller masses by improving triggers and experimental techniques.

A possible way to reduce the mass threshold in trigger and deal with the huge rate of selected events at low masses $\left(m_{j j}<1.6 \mathrm{TeV}\right)$ is to reconstruct, select, and record events in a compact form by the high-level trigger in a technique called either data scouting (CMS [10]) or trigger-object level analysis (ATLAS [11]). Doing so, the trigger threshold can be significantly reduced and the search becomes sensitive to masses down to $400-600 \mathrm{GeV}$.

Another trick to explore the low-mass regime is to look for events where the hypothetical resonance is produced together with an energetic ISR jet, that can be used to trigger the event. 

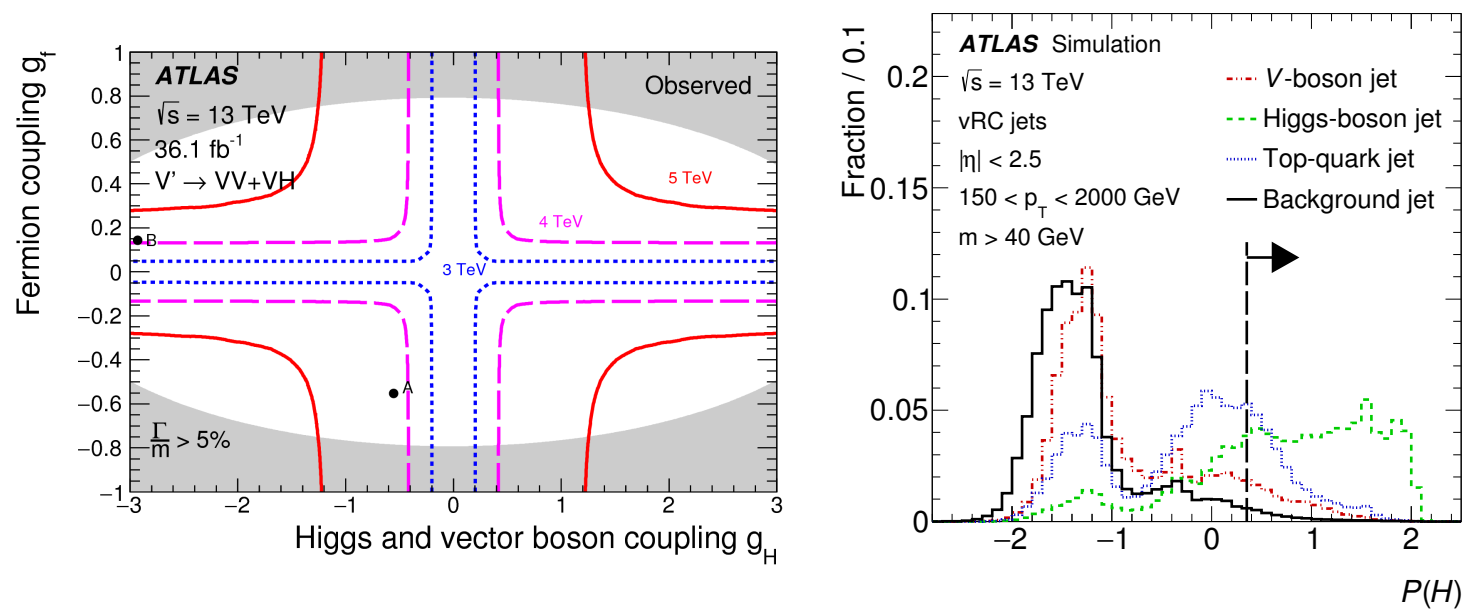

Figure 3: Left: Observed 95\% CL exclusion contours in the $g_{H}, g_{f}$ HVT parameter space for resonances of mass 2, 3, 4, or $5 \mathrm{TeV}$ for the combination of $\mathrm{VV}$ and $\mathrm{VH}$ channels [16]. The areas outside the curves are excluded. The gray area indicates parameter regions for which $\Gamma / \mathrm{m}>5 \%$. Right: an example of the discriminant function $\mathrm{P}$, based on a deep neural network, for the Higgs-tagger [17]. Background jets are taken from simulated multi-jet events. The dashed vertical line represents the applied tagging selection.

The resonance is produced with sufficiently high transverse momentum that its decay products are merged into a single jet with a two-prong substructure. The signal is then identified as a peak over a smoothly falling background in the distribution of the invariant mass of the jet, using novel jet substructure techniques. The sensitivity is now mainly limited by the jet mass reconstruction (either the two prongs are too close or too far each other) and can go down to $50 \mathrm{GeV}$ [12].

The last approach to extend the mass range to lower values is to include extra requirements, either in the jet flavor of the decay products of the resonance [13, 14] or looking for additional activity in the event, like for instance the presence of leptons with high transverse momentum [15].

In none of the searches described above, significant deviations from the SM are observed. A nice summary of the different experimental results is shown in Fig. 2. It can be noticed that we are now able to cover a large fraction of the mass spectrum and limits from the LHC are better than previous experiments at every mass.

\subsection{Dibosons and substructures}

When the resonance decays in bosons (W, Z, or Higgs) or in top quarks, the search becomes more challenging. The reason is that particles produced from the decay tend to occupy small spacial regions. For instance, in case of hadronic decays, bosons produced in the decay of TeVscale resonances are highly boosted, and therefore are reconstructed as a single large jet. The information from the different detectors can be then used to exploit the substructure of the jet, which must appear as a two- or three-prong decay, as opposed to the QCD background jets which have a single prong structure. Further care must be given to the b-tagging in the case of the $\mathrm{Z}$ and Higgs boson decays to $\mathrm{b}$ quarks.

ATLAS and CMS experiments have both an extensive research program to search for diboson resonances. ATLAS has recently published a combination of several channels [16] in specific 

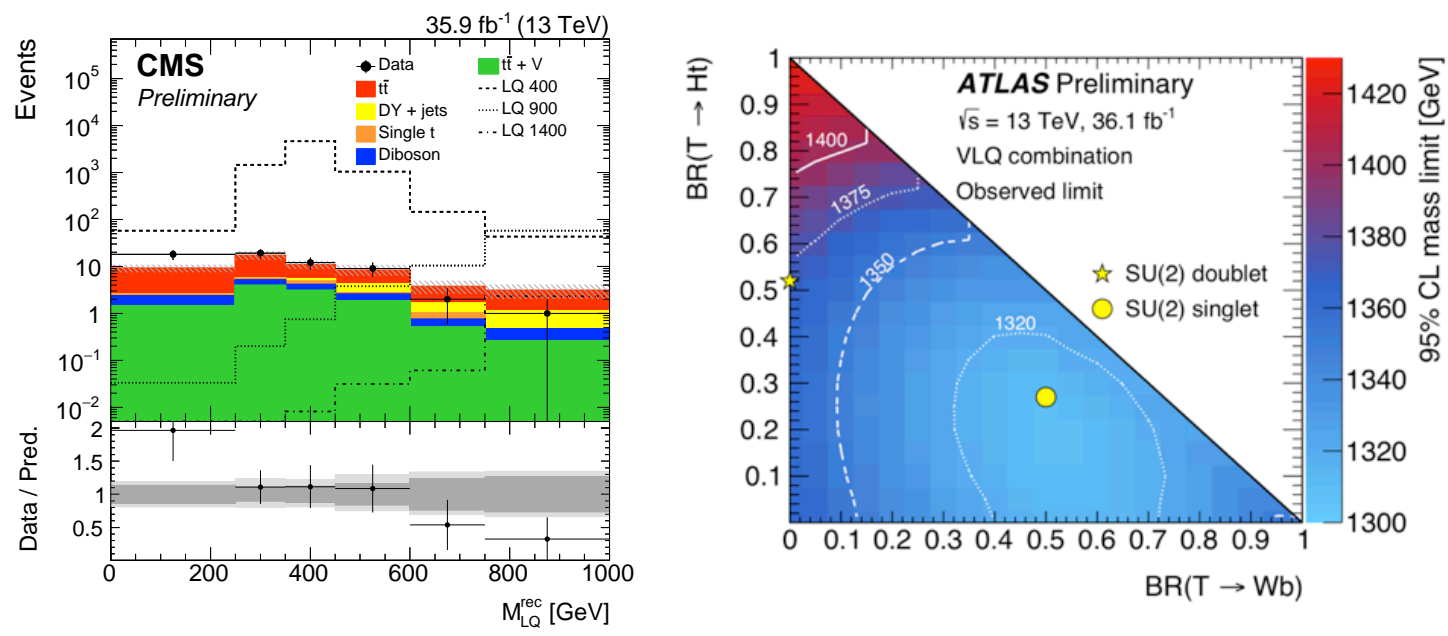

Figure 4: Left: distributions for reconstructed mass of the leptoquark decaying to $\mu\left(M_{L Q}^{r e c}\right)$ after applying the full selection [21]. Right: observed lower limits at 95\% CL on the mass of the T vector like quark, resulting from a combination of several different searches, as a function of branching ratio assuming $\mathrm{B}(\mathrm{T} \rightarrow \mathrm{Ht})+$ $\mathrm{B}(\mathrm{T} \rightarrow \mathrm{Zt})+\mathrm{B}(\mathrm{T} \rightarrow \mathrm{Wb})=1[23]$.

benchmark models, where different resonances are expected to decay in final states with $\mathrm{W}, \mathrm{Z}$, and Higgs bosons. In Fig. 3 the limits are obtained within the heavy vector triplet model, where a triplet of colorless vector bosons is introduced with zero hypercharge. They are nearly degenerate in mass and correspond to two charged $\mathrm{W}^{\prime}$, and a neutral Z', collectively denoted by $\mathrm{V}$ '. The parameters $g_{H}$ and $g_{f}$ correspond to the coupling strengths between the triplet field $\mathrm{W}$ and the fermion and Higgs fields, respectively. The results in different decay modes can be then combined, deriving constraints on the couplings.

The substructures techniques can be further boosted by improving the performance via new advances in machine learning to better distinguish various types of jets. In Fig. 3 an example by the ATLAS experiment is shown. A multi-class deep neural network is trained to identify the most likely parent particle of the jets, distinguishing between four categories: V-boson (W or Z), Higgsboson, top-quark, and background jets [17]. There are many ongoing efforts to expand the use of these techniques and to further improve the identification of the structure of the jets via imaging (e.g. $[18,19])$.

\section{New leptons and quarks}

Some of the most significant deviations from the predictions of the SM have been observed in measurements of rare decays of B mesons. The values of the ratios $R_{D}$ and $R_{D}^{*}$, defined as ratios of the branching fractions for $B^{0} \rightarrow D^{(*)} \tau \nu$ to those for $B^{0} \rightarrow D^{(*)} \mu \nu$ show a combined significance of about four standard deviations. Moreover, the ratio of the branching fraction of $B^{+} \rightarrow K^{+} \mu^{+} \mu^{-}$to $B^{+} \rightarrow K^{+} e^{+} e^{-}$as measured by the LHCb collaboration, shows a departure from lepton universality by 2.6 standard deviations. The existence of leptoquarks (LQs) with masses at the $\mathrm{TeV}$ scale has been proposed as a possible explanation of these deviations. Leptoquarks are hypothetical particles that decay to SM quarks and leptons and can be identified with a lepton and a jet, whose invariant mass peaks at the leptoquark mass. As for leptons and quarks, there exist three 
generation of leptoquarks, which can be identified by the flavor of the decay products. In addition, the leptoquarks can be produced in pairs, thus allowing a selection based on the request of a pair of jets and a pair of leptons.

Recently, the CMS experiment produced new constraints on the mass of these hypothetical first and third generation leptoquarks [20, 21, 22]. To give an example, the production in pairs of third generation LQs decaying in $t \mu$ can be studied [21]. As for other LQ analyses, the most significant discriminating variables are the reconstructed invariant mass of the LQ (which should peak at the mass of the LQ, see Fig. 4) and the scalar sum of the transverse momentum of the physics objects in the event. No sign of new physics have been observed and pair-produced scalar LQs decaying exclusively into a top quark and a muon have been excluded below masses of 1420 $\mathrm{GeV}$.

In order to solve the hierarchy problem vector-like quark (VLQ) models have been proposed. These new heavy partners of top would take part in loops and fix the quadratic divergence to the Higgs boson mass. A combination of searches of a up-type T VLQ with charge $=2 / 3$, decaying into $\mathrm{Wb}, \mathrm{Zt}$, or $\mathrm{Ht}$, and a down-type quark $\mathrm{B}$ with charge=-1/3, decaying into $\mathrm{Wt}, \mathrm{Zb}$, or $\mathrm{Hb}$, have been recently presented by the ATLAS experiment [23]. The production of these particles in pairs allows for a variety of different topologies, which are explored by several complementary analyses, involving the decay of the $\mathrm{W}, \mathrm{Z}$, and $\mathrm{H}$ bosons in fully leptonic modes (including invisible $\mathrm{Z}$ decays in $v v$ ) and fully hadronic modes in bb and jetjet. The result of this combination is shown in Fig. 4. It can be noticed that the $95 \%$ confidence limits in mass for the T VLQ range beween 1300 and $1400 \mathrm{GeV}$, depending on the branching ratios in the three possible decay modes. The equivalent limits for the B quark range between 1000 and $1300 \mathrm{GeV}$.

\section{Long-lived signatures}

Long-lived (LL) and unconventional exotic particles with striking signatures are predicted by many extensions of the SM, like several SUSY scenarios (e.g. Gauge Mediated Supersymmetry Breaking or scenarios with mass-degenerate gauginos) and in models where there is an hidden sector that couples very weakly to visible sector fields. These new long-lived particles would show up in several different topologies in detectors: delayed photons, displaced track vertexes, displaced jets and leptons, disappearing tracks, almost stable charged particles, particles stopped in the detector, and emerging jets. The LHC detectors were not originally intended to detect particles that decay further than a few centimeters from the beamline and the original focus for displaced signature was only on detecting jets containing bottom or charm quarks, which decay a few hundred micrometers from the beam axis. Today, there is a pletora of searches and the LL physics program is in rapid expansion. Among all these results, we can discuss a few, focusing on the most recent ones.

Since these topologies look sensibly different from particles produced by the SM processes, it is very likely to confuse them as noise. Then, their identification implies a very good knowledge of detector performance, resolutions and electronic noise. After several years of data taking, now LHC experiments and their detector simulations reached a level of understanding to allow for the search of many of these processes. 


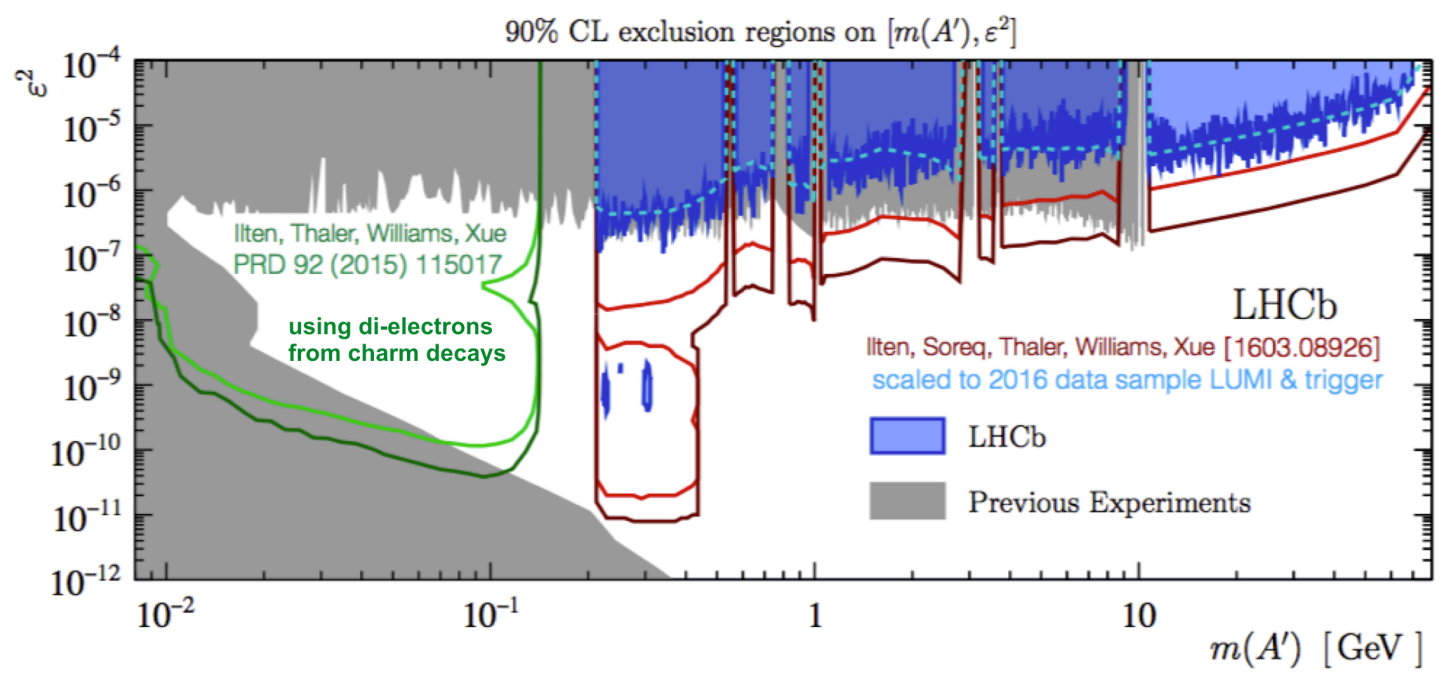

Figure 5: Regions of the $\left[\mathrm{m}\left(\mathrm{A}^{\prime}\right), \varepsilon^{2}\right]$ parameter space excluded at $90 \%$ CL by the prompt-like and displaced A' search compared to the best existing limits [26]. The red and green curves show the limits expected at the end of LHC Run 3.

CMS has recently published a search, based on $13 \mathrm{TeV}$ p-p collisions, for LL charged particles that decay within the detector and produce the signature of a disappearing track [24]. The signature corresponds to an isolated track with missing hits in the outer layers of the silicon tracker, tiny energy in associated calorimeter deposits, and no associated hits in the muon detectors. No deviations from the SM are observed and limits in the context of the anomaly-mediated supersymmetry breaking model are set. At $95 \%$ confidence level, charginos with masses below about $700 \mathrm{GeV}$ are excluded for a lifetime of about 3-7 ns.

Another interesting and novel analysis by the CMS experiment is the search for emerging jets [25]. The scenario corresponds to the production of a new heavy mediator that decays to a light quark and a new fermion, called a dark quark. This new quark can then form long-lived dark hadrons via a parton shower which finally decay to SM particles, thus producing a striking signature consisting in a jet with many displaced vertexes. The search excludes mediator particles with masses between 400 and $1250 \mathrm{GeV}$ for dark hadron decay lengths between 5 and $225 \mathrm{~mm}$.

The presence of dark forces may explain how dark matter particles interact with SM particles. An interesting dark-force scenario involves a massive dark photon A', whose coupling to the electromagnetic current is suppressed relative to that of the ordinary photon by a factor $\varepsilon$. A' can be produced at $\mathrm{p}-\mathrm{p}$ collisions since it mixes with the SM photon via a kinetic term proportional to $\varepsilon$. The $\left[\mathrm{m}\left(\mathrm{A}^{\prime}\right), \varepsilon^{2}\right]$ plane can be explored with an inclusive search for $\mathrm{A}^{\prime} \rightarrow \mu^{+} \mu^{-}$decays with the LHCb experiment [26]. Depending on the strength of $\varepsilon, A^{\prime}$ can be stable or long-lived. Then, by searching for displaced $\mu^{+} \mu^{-}$signatures, very small values of $\varepsilon$ are accessible. In Fig. 5 the most recent results from $\mathrm{LHCb}$ are shown in blue. In can be noticed that at intermediate masses $\mathrm{LHCb}$ limits are competitive with ones obtained at B factories, while for masses $>10 \mathrm{GeV}$ the constraints placed are the most stringent to date. The displaced search has been also able to put limits for $\varepsilon^{2} \sim 10^{-9}$ at masses of the order of a few hundreds $\mathrm{MeV}$. In the same plot the expected limits, corresponding to the Run 3 statistics, are also displayed, showing that $\mathrm{LHCb}$ will provide the best 
constraints for a large fraction of the phase space.

Recently, also ATLAS has presented searches for the dark photon in Higgs decays [27, 28]. There are two different signatures explored. The first one corresponds to a Higgs boson, which mixes with a dark Higgs, decaying into two dark photons and producing a four lepton final state. The second one corresponds to a Higgs boson decaying into two SM Z bosons, where one of the two mixes with a long-lived dark photon, producing a displaced dilepton signature. In both cases, the data are found to be consistent with SM background predictions. The prompt analysis provides upper limits on the branching ratio of the Higgs to $\mathrm{Z}$ plus dark photon decay mode of the order of $0.1 \%$ and on the branching ratio of the Higgs to two dark photons mode of the order of $0.01 \%$. The displaced search sets upper limits on cross-section times branching fraction as a function of the lifetime, for dark-photon masses in the range $20-60 \mathrm{GeV}$, which can be also translated into constraints on the Z-dark photon mixing parameter.

\section{Dark matter searches}

The existence of so-called dark matter (DM) comes from the fact that cosmological and astrophysical observations show that the majority of the matter is non-luminous. There are different models to explain the origin of the DM. Among them, there is the scenario where the candidate for DM is a weakly interacting massive particle. In the search for DM different experimental techniques are used. In indirect searches, space-based and ground-based observatories should detect secondary particles from the annihilations of dark matter candidates. Direct detection experiments search for the scattering of DM on SM particles (nuclei and electrons) in low background and deep underground environments. Finally, at the LHC experiments, DM can be produced in pairs in p-p collisions.

Since DM goes basically unseen in the LHC detectors, the events of interest are identified using additional particles. Either jet, $\gamma, \mathrm{W}, \mathrm{Z}, \mathrm{H},(t) \bar{t}$, or $(b) \bar{b}$, must be produced in association with the DM, in order to tag the event. The presence of the recoiling undetected DM is then inferred from the imbalance of the event in the transverse plane, quantified by the missing transverse momentum $\left(E_{T}^{\text {miss }}\right)$.

The main complication of these searches is the background discrimination, given that $E_{T}^{\text {miss }}$ look very similar for signal and background events. In addition, the cross sections of background processes are orders of magnitude larger than the DM one and some SM event topologies are impossible to be distinguished from signal. For instance, $\mathrm{Z}(\rightarrow v v)+\mathrm{jet}$ events look identical to the signal events in the DM+jet search. To properly measure the SM contributions, the analysis is performed in several different event categories, in order to have signal enriched categories and control samples, which are used to model each single background contribution. A global fit is then run to determine the amount of signal and the normalization and shape of the background components (see for example [29]).

The results of the search are interpreted in terms of simplified DM models assuming a vector, axial-vector, scalar, or pseudoscalar mediator, which decays into a pair of fermionic DM particles and interacts with SM particles. In these models there are four free parameters: the coupling strength of the mediator interaction with DM $\left(g_{D M}\right)$, the coupling strength of the mediator interaction with SM particles ( $g_{q}$ and $g_{l}$, for quarks and leptons, respectively), the mass of the mediator 

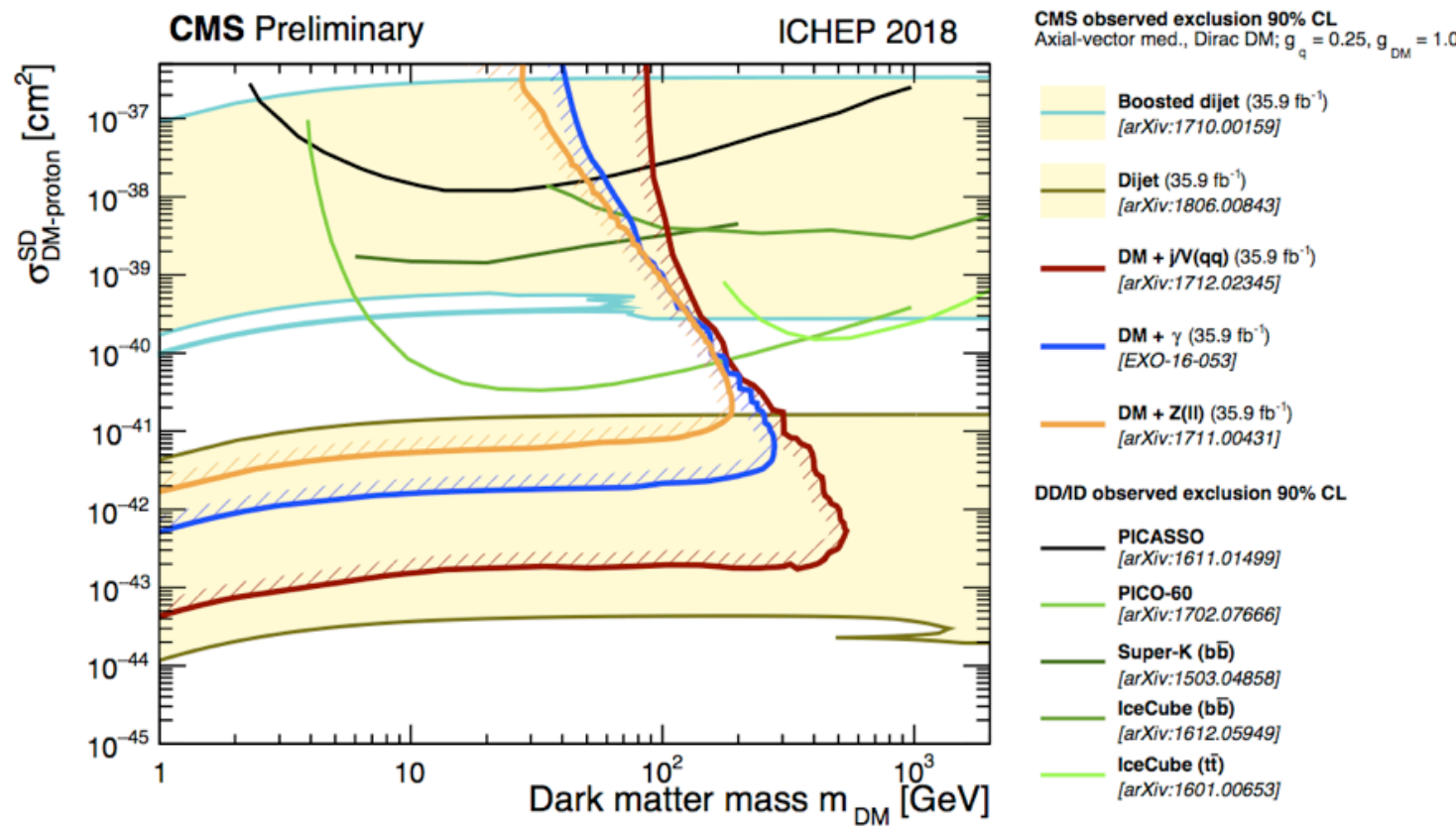

Figure 6: A comparison of CMS results to the $m_{D M}-\sigma_{S D}$ (spin dependent) plane [9]. The limits are shown at $90 \% \mathrm{CL}$. The CMS contour is for an Axial-vector mediator, Dirac DM and couplings $g_{q}=0.25$ and $g_{D M}=1.0$. The SD exclusion contour is compared with limits from PICASSO and PICO experiments, the IceCube limit for the $t \bar{t}, b \bar{b}$ annihilation channels, and the Super-Kamiokande limit for the $b \bar{b}$ annihilation channel.

$\left(M_{m e d}\right)$, and the mass of the dark matter particle $\left(m_{D M}\right)$. Usually, constraints from the measurements are presented after fixing two of these four parameters and scanning the other two. Since the mediator couples to DM and to SM particles, it can decay also to a pair of jets and therefore can be observed as a dijet resonance. Results from heavy resonance searches can be then translated into limits and added to the constraints on the four parameters discussed before.

The limits obtained using the simplified DM models may be compared to the results from direct and indirect DM detection experiments, which are usually expressed as upper limits on the DM-nucleon scattering cross sections. An example of the constraints from $\mathrm{DM}+\mathrm{jet}, \mathrm{DM}+\gamma$, $\mathrm{DM}+\mathrm{Z}$ and dijet searches, assuming an axial-vector mediator, are shown in Fig. 6 and compared to constraints from direct detection. The best constraints for spin- 0 mediators are instead obtained with $\mathrm{DM}+(t) \bar{t}$ or $(b) \bar{b}$ searches, since the coupling of the mediator is in this case proportional to the quark mass [30].

\section{Conclusions}

The outstanding performance of the LHC led to more than $100 \mathrm{fb}^{-1}$ of accumulated $13 \mathrm{TeV}$ data by summer of 2018, exceeding the expectations. This large amount of data has allowed for a multitude of searches for beyond Standard Model scenarios involving striking new signatures, like heavy resonances, new leptons and quarks, long-lived particles, and dark matter. Unfortunately no excess of events has been observed and the measurements are consistent with the Standard Model. 
While moderate improvements for extremely high mass searches are expected in future, there are several new approaches, targeting low and intermediate mass regions and using novel experimental techniques, which will explore new territories during next years.

\section{References}

[1] G. Aad et al. [ATLAS Collaboration], JINST 3, S08003 (2008).

[2] S. Chatrchyan et al. [CMS Collaboration], JINST 3, S08004 (2008).

[3] A. A. Alves, Jr. et al. [LHCb Collaboration], JINST 3, S08005 (2008).

[4] CMS public: https://twiki.cern.ch/twiki/bin/view/CMSPublic/LumiPublicResults.

[5] ATLAS public: https://twiki.cern.ch/twiki/bin/view/AtlasPublic/LuminosityPublicResultsRun2.

[6] ATLAS public: https://twiki.cern.ch/twiki/bin/view/AtlasPublic/ExoticsPublicResults.

[7] CMS public: http://cms-results.web.cern.ch/cms-results/public-results/publications/EXO/index.html, http://cms-results.web.cern.ch/cms-results/public-results/publications/B2G/index.html.

[8] LHCb public: http://lhcbproject.web.cern.ch/lhcbproject/Publications/LHCbProjectPublic/Summary_QEE.html.

[9] https://twiki.cern.ch/twiki/bin/view/CMSPublic/SummaryPlotsEXO13TeV.

[10] A. M. Sirunyan et al. [CMS Collaboration], JHEP 1808, 130 (2018).

[11] M. Aaboud et al. [ATLAS Collaboration], Phys. Rev. Lett. 121, no. 8, 081801 (2018).

[12] A. M. Sirunyan et al. [CMS Collaboration], JHEP 1801, 097 (2018).

[13] M. Aaboud et al. [ATLAS Collaboration], Phys. Rev. D 98, 032016 (2018).

[14] CMS Collaboration, CMS-PAS-EXO-17-024, https://cds.cern.ch/record/2628756.

[15] ATLAS collaboration, ATLAS-CONF-2018-015, https://cds.cern.ch/record/2621126.

[16] M. Aaboud et al. [ATLAS Collaboration], Phys. Rev. D 98, 052008 (2018).

[17] M. Aaboud et al. [ATLAS Collaboration], Phys. Rev. D 98, 092005 (2018).

[18] ATLAS Collaboration, ATL-PHYS-PUB-2017-017, http://cdsweb.cern.ch/record/2275641.

[19] CMS Collaboration, CMS-DP-2017-027, https://cds.cern.ch/record/2275226.

[20] CMS Collaboration, CMS-PAS-EXO-17-009, https://cds.cern.ch/record/2628477.

[21] CMS Collaboration, CMS-PAS-B2G-16-027, https://cds.cern.ch/record/2621420.

[22] CMS Collaboration, CMS-PAS-EXO-17-016, https://cds.cern.ch/record/2628542.

[23] M. Aaboud et al. [ATLAS Collaboration], Phys. Rev. Lett. 121, 211801 (2018).

[24] A. M. Sirunyan et al. [CMS Collaboration], JHEP 1808, 016 (2018).

[25] CMS Collaboration, CMS-PAS-EXO-18-001, https://cds.cern.ch/record/2625123

[26] R. Aaij et al. [LHCb Collaboration], Phys. Rev. Lett. 120, no. 6, 061801 (2018).

[27] M. Aaboud et al. [ATLAS Collaboration], JHEP 1806, 166 (2018)

[28] ATLAS Collaboration, CERN-EP-2018-173, https://cds.cern.ch/record/2633725.

[29] M. Aaboud et al. [ATLAS Collaboration], JHEP 1801, 126 (2018).

[30] CMS Collaboration, CMS-PAS-EXO-16-049, https://cds.cern.ch/record/2311235. 